\title{
Acute urinary retention due to cystic echinococcosis: A case report
}

\author{
Volkan Izol, MD, FEBU; Alper Eken, MD; I. Atilla Aridogan, MD; ${ }^{*}$ Soner Koltas, PhD; Zuhtu Tansug, MD*
}

*Cukurova University, Medical Faculty, Department of Urology, Adana, Turkey; †Cukurova University, Medical Faculty, Department of Parasitology, Adana, Turkey

Cite as: Can Urol Assoc J 2012;6(5):E192-E194. htrp://dx.doi.org/10.5489/cuai.11154

\begin{abstract}
Urinary hydatidosis is very rare and represents $2 \%$ to $4 \%$ of all cases of cystic echinococcosis. We present a case of a 21 -yearold man with symptoms of frequency, urinary retention and reduced force in urinary stream due to cystic echinococcosis. Anti-Echinococcus granulosus antibodies were determined by echinococcosis western blotting, and pelvic computed tomography revealed a $11 \times 14 \times 10-\mathrm{cm}$ retrovesical homogeneous cystic mass. The patient underwent surgical intervention; the cystic mass and retrovesical cavity were removed. After a 3-year follow-up period, there was no episode of lower urinary tract symptoms. This case illustrates that cystic echinococcosis should be considered in every case of cystic mass, especially in endemic countries, such as Turkey.
\end{abstract}

\section{Introduction}

Cystic echinococcosis (CE) of Echinococcus granulosus develops in internal organs (mainly liver and lungs) of humans and other intermediate hosts with unilocular fluid-filled bladders. The definitive hosts of $E$. granulosus are carnivores, such as dogs and wolves, which are infected by the ingestion of offal containing hydatid cysts with viable protoscoleces. After ingestion, the protoscoleces evaginate, attach to the canine intestinal mucosa and develop to adult stages. Sexual maturity (length of 3 to $6 \mathrm{~mm}$ ) is reached 4 to 5 weeks later. Eggs or gravid proglottids are shed in the feces. Following ingestion by a human or ungulate intermediate host (sheep, goats, pigs, cattle, horses, and camels), an oncosphere larva is released from the egg. The larvae then penetrate into the lamina propria and are transported passively through blood or lymph vessels to the liver, lungs or other organs where the oncosphere larvae develop into hydatid cysts (metacestode larvae). It has parasite-derived layers: an inner nucleated germinal layer and an outer acel- lular laminated layer surrounded by a host-derived fibrous capsule. Brood capsules and protoscoleces bud from the germinal membrane. The range of intermediate host species depends on the infecting strain of E. granulosus, regional or local differences in the availability of various intermediate host species, and other factors. ${ }^{1}$

CE is an important parasitic disease caused by the dog tapeworm $E$. granulosus. CE is extremely widespread in eastern and southern Europe, the Middle East, North Africa and South America. ${ }^{2}$ Human CE is a serious public health problem in Turkey. ${ }^{3}$ The Adana province, in the southern part of the Mediterranean region of Turkey, has a high number of surgical cases of CE. Several published reports have investigated CE. ${ }^{4}$

We present a case of urinary retention due to retrovesical $\mathrm{CE}$ and review the diagnosis and surgical treatment.

\section{Case report}

A 21-year-old man presented with a 1-year history of frequency, dribbling, constipation and reduced force of the urinary stream. He was admitted to the Urology Department of the University of Cukurova with acute urinary retention. His white blood cell count was $10.500 / \mathrm{mm}^{3}$, and eosinophilic leucocytes constituted $6.68 \%$ of his total white blood cell count. About $2000 \mathrm{~mL}$ of urine was drained by an indwelling catheter. Cysto-urethroscopy revealed that the prostatic urethra was long; it was not possible to evaluate the bladder and high bladder neck. On digital rectal examination, there was a soft-tissue mass in place of the prostate. Pelvic computerized tomography $(\mathrm{CT})$ was planned, and the procedure was terminated. Pelvic CT demonstrated a $11 \times 14 \times 10-\mathrm{cm}$ retrovesical homogeneous cystic mass and displacement of the urinary bladder anteriorly and superiorly by the cyst. The right seminal vesicle was identified with difficulty, while the left seminal vesicle was not visualized (Fig. 1). The pelvic CT suggested that the cyst had arisen from the seminal vesicle or prostate gland. With the patient under general anesthesia, 


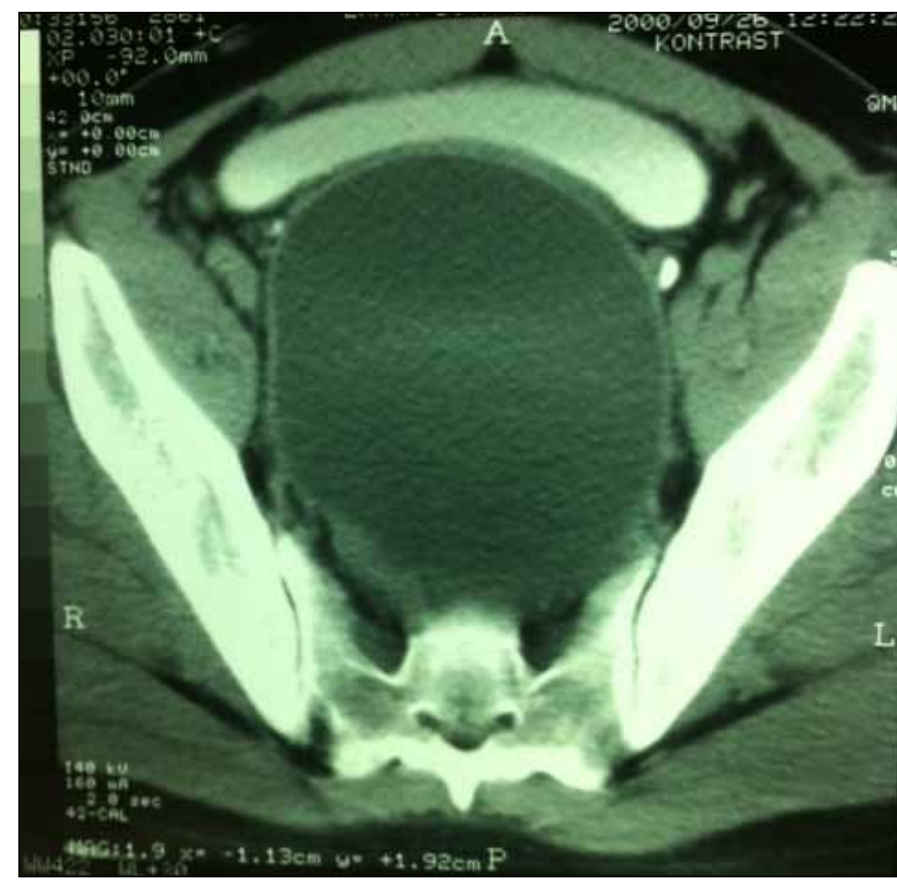

Fig. 1. Retrovesical homogeneous cystic mass and displacement of the urinary bladder anteriorly and superiorly by the cyst was detected on pelvic computed tomography.

$875 \mathrm{~mL}$ of clear fluid was drained from the cyst by suprapubic aspiration. The impression of the cyst in the bladder wall disappeared during cystourethroscopy. The cytology and culture of the cyst fluid were negative for tuberculosis. After 2 weeks, a control CT was performed and a retrovesical cystic mass with a germinative membrane-like hydatid cyst was detected (Fig. 2). The indirect hemagglutination test (IHA, Fumouze, France) for cystic echinococcosis was negative. Anti-E. granulosus antibodies were detected by Echinococcosis western blotting (Euroimmun, Germany), which was performed according to the protocols established by the manufacturer.

Laparotomy and cyst excision were planned and performed. The anterior wall of the cyst was incised, and $800 \mathrm{~mL}$ of inflamed fluid was drained. At the end of the aspiration, a $10 \times 5-\mathrm{cm}$ germinative membrane was excised. The cavity of the cyst was later irrigated with $30 \%$ sodium chloride and aspirated. Two drains were placed in the cyst and retrovesical cavity. An 800-mg/day albendozole regimen was initiated 3 days before surgery and continued after the surgery for 3 months. The drains were removed on the 7th and 8th days, and the patient was discharged. The pelvic CT was normal by the 3rd month postoperatively (Fig. 3). No complication or recurrence was observed in the 3-year follow-up period after surgery.

\section{Discussion}

Although CE has been recognized in Turkey since 1861, a

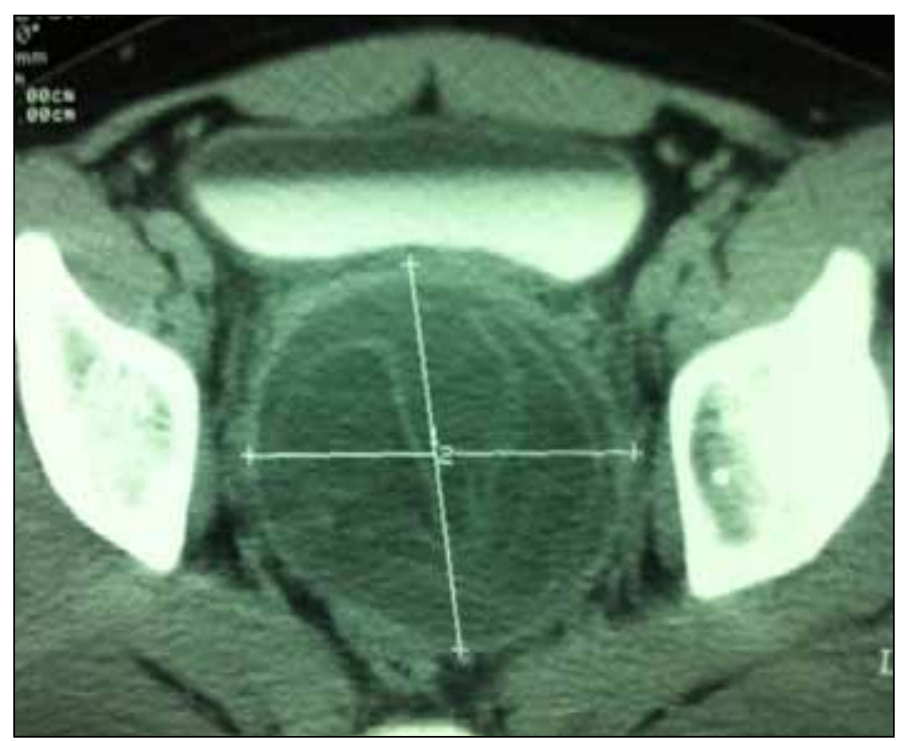

Fig. 2. After the aspiration, a germinative membrane-like hydatid cyst was seen on computed tomography (CT). Note the difference from the first CT.

consistent state policy for disease prevention and control has not been developed, and cystic echinococcosis continues to be a major health problem in Turkey. ${ }^{5}$ Although not clearly defined, isolated pelvic CE are exceedingly rare around the world. Within the urinary tract, the kidneys are the most commonly affected organs, but CE of the bladder, prostate, seminal vesicles and testis has also been reported.6,7 In a few cases, retrovesical CE has been reported in Turkey. ${ }^{8,9}$

Our patient was 21 years old. His father is a farmer, and he lives in close contact with sheep and dogs. The reason for the delayed diagnosis is that a CE usually grows very slowly, taking years to reach an appreciable size; it has an asymptomatic course until it attains full growth. There are no specific clinical symptoms and signs that will enable accurate diagnosis of urinary tract CE. ${ }^{10}$ The symptoms of CE are generally the same as those of other space-occupying lesions, except for hydaturia caused by rupture of the cyst into the collecting system of the urinary tract. The symptoms of our case were non-specific, including lumbar or abdominal pain, malaise, nausea and vomiting, as well as irritative and obstructive lower urinary tract symptoms. Laboratory tests for eosinophilia, the casoni skin test and IHA are not reliable. However, the detection of anti-E. granulosus antibodies determined by echinococcosis western blotting has been very valuable in the past few decades. Among the radiological investigations, CT is more sensitive and accurate. Although the first CT was not diagnostic of CE in our patient, the second CT, which was performed after suprapubic aspiration, showed multi-vesicular cysts of mixed density. CE is usually unilocular. As the cyst matures, it transforms itself into a larger multivesicular and occasionally multilocular cyst containing numerous daughter cysts. ${ }^{11}$ This is probably why we could not detect the disease in the first CT. 
Izol et al.

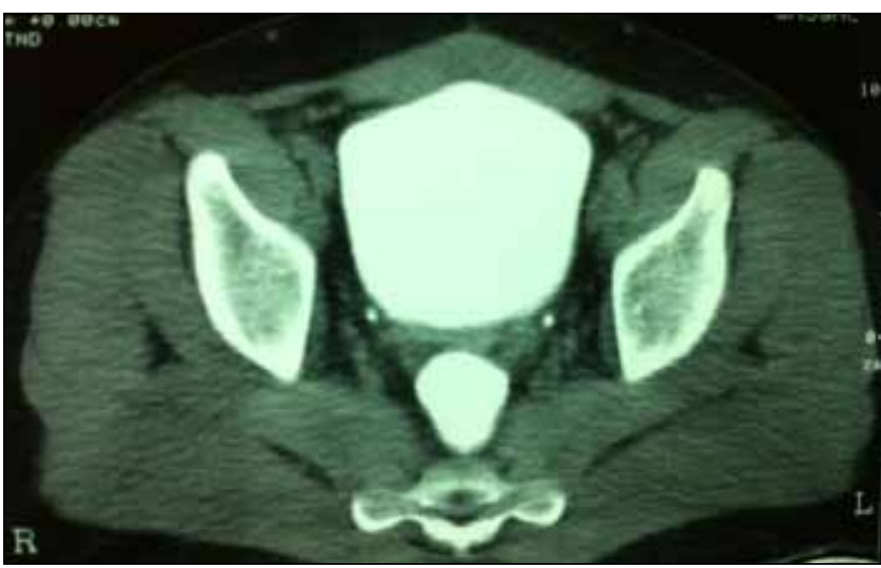

Fig. 3. The pelvic computed tomography in the third month postoperatively.

Surgical treatment of CE requires particular care with respect to the cyst contents. If a patient with renal CE has a solitary kidney, marsupialization or percutaneous drainage should be performed. If drainage is performed, the contents of the cyst should initially be aspirated, and the cyst should be filled with a scolecidal agent, such as $30 \%$ sodium chloride, $2 \%$ formalin or $1 \%$ iodine, for about 5 minutes to kill the germinal portions of the cyst. ${ }^{12}$ However, anti-helminthic agents do not always sterilize the contents of a cyst in all instances and have significant side effects. Because of the danger of rupture and spillage of the highly antigenic contents and the risk of anaphylaxis, percutaneous aspiration of CE is not usually recommended. In our case, we did not suspect CE in the first place and aspirated the cyst.

We present a rare case of a CE involving the retrovesical region of a 21-year-old man. Urinary retention was the initial symptom. Both imaging and clinical evaluation revealed a substantial retrovesical cystic mass. The histopathological report was CE of the seminal vesicles. CE of the urinary tract is relatively uncommon and is likely to cause considerable diagnostic difficulties for clinicians and radiologists; therefore, it should be kept in mind in the differential diagnosis of space-occupying lesions of the urinary tract. These cysts can grow enormously, occupy the entire pelvis, and can cause urinary tract symptoms as observed in our case. Although the preoperative meticulous imaging is necessary, histopathologic diagnosis after surgical removal is mandatory especially in endemic regions.

\section{Conclusion}

This case of CE of the seminal vesicles is one of the rare entities reported in the literature. The diagnosis of CE should be kept in mind with patients who have cystic lesions in the retrovesical region.

Competing interests: None declared.

This paper has been peer-reviewed.

\section{References}

1. Eckert J, Deplazes P, Craig PS, et al. Echinococcosis in animals: clinical aspects, diagnosis and treatment. In: Eckert J, Gemmell M, Meslin FX, Pawlowski Z, editors. WHOI/OIE manual on echinococcosis in humans and animals: a public health problem of global concern. Paris: World Organization for Animal Health; 2001:72-99.

2. Symbas PN, Aletras H. Hydatid disease of the lung. In: Shields TW, editor. General thoracic surgery, 4th Edition. Philadelphia: Williams \& Wilkins; 1994:1021.

3. Yazar S, Yaman 0, Cetinkaya F, et al. Cystic echinococcosis in Central Anatolia, Turkey. Saudi Med J 2006;27:205-9.

4. Yazar S, Ozkan AT, Hökelek M, et.al. Cystic echinococcosis in Turkey from 2001-2005. Türkiye Parazitol Derg 2008;32:208-20.

5. Altintas N. Past to present: echinococcosis in Turkey. Acta Tropica 2003;85:105-12. http://dx.doi. org/10.1016/S0001-706X(02)00213-9

6. Safioleas $M$, Stamatakos M, Zervas A, et al. Hydatid disease of the seminal vesicle: A rare presentation of hydatid cyst. Int Urol Nephrol 2006;38:287-9. http://dx.doi.org/10.1007/s1 1255-006-6652-9

7. Angulo JC, Escribano J, Diego A, et al. Isolated retrovesical and extrarenal retroperitoneal hydatidosis: clinical study of 10 cases and literature review. J Urol 1998;159:76-82. http://dx.doi.org/10.1016/ S0022-5347(01)64016-3

8. Emir L, Karabulut A, Balci U, et al. An unusual cause of urinary retention: a primary retrovesical echinococcal cyst. Urology 2000;56:856. http://dx.doi.org/10.1016/S0090-4295(00)00759-7

9. Uygur MC, Gülerkaya B, Karakoc A, et al. A Solitary Hydatid Cyst Of The Retrovesical Region. Int Urol Nephrol 1999;31:45-7. http://dx.doi.org/10.1023/A:1007167622265

10. Ozbey I, Aksoy Y, Bicgi 0 , et al. Hydatid disease of the urinary tract: review of the management of 9 cases. Int Urol Nephrol 2001;33:329-34. http://dx.doi.org/10.1023/A:1015209106436

11. Rastogi, R. Giant Solitary echinococcal cyst of the seminal vesicle: An uncommon cause of retention of urine. Saudi I Kidney Dis Transpl 2008;19:799-801.

12. Sengör $F$, Narter $F$, Erdogan $K$, et al. Echinococcal cyst of the kidney: A review of the management of 11 cases. Int Urol Nephrol 1996;28:289. http://dx.doi.org/10.1007/BF02550488

Correspondence: Dr. Volkan Izol, University of Cukurova, Faculty of Medicine, Department of Urology, 01330, Adana, Turkey; fax: +90 322 4573072; volkanizo|@yahoo.com 